\title{
Diseño y construcción de un pico-satélite educativo CanSat tipo rover denominado EagleSat V2.1
}

\author{
Design and construction of an educational CanSat pico-satélite rover type called \\ EagleSat V2.1
}

MANCILLA-CEREZO, Josue †*, PALACIOS-GARCIA, Ana Cristina, PÉREZ-LOPEZ, Adriana y JIMENEZ-GONZALEZ, Diane Grace

Instituto Tecnológico Superior de Tepeaca

Instituto Tecnológico Superior de Teziutlan

ID $1^{\text {er }}$ Autor: Josue, Mancilla-Cerezo / ORC ID: 0000-0002-9659-3677, CVU CONACYT ID: 457562

ID $1^{\text {er }}$ Coautor: Ana Cristina, Palacios-Garcia / ORC ID: 0000-0003-3138-9699, CVU CONACYT ID: 946392

ID $2^{\mathrm{do}}$ Coautor: Adriana, Pérez-Lopez / ORC ID: 0000-0003-3712-400X, CVU CONACYT ID: 478724

ID $3^{\text {er }}$ Coautor: Diane Grace, Jimenez-Gonzalez / ORC ID: 0000-0003-3072-7107, CVU CONACYT ID: 1000644

DOI: $10.35429 /$ JOIE.2019.12.3.17.23

Recibido 13 de Agosto, 2019; Aceptado 30 de Noviembre, 2019

\begin{abstract}
Resumen
Se describe como se diseñó y construyó un pico-satélite CanSat tipo rover para concursar en el Cuarto Concurso Nacional de Pico-Satélites Educativos CanSat. Alumnos de Ingeniería en Tecnologías de la Información y Comunicaciones con el apoyo de docentes de dicha carrera diseñaron y construyeron el pico-satélite denominado EagleSat V2.0. Utilizando la metodología del modelo "V", se conceptualizó la misión la cual fue él envió de datos de: temperatura interna y externa, presión, humedad relativa, altitud, longitud, latitud, nivel de batería, vibración y aceleración por medio de telemetría a una estación terrena; tomar video y el retorno al punto de partida mediante un vehículo tipo rover; se especificaron los requerimientos y la arquitectura de todas las etapas del EagleSatV2.1. Partiendo de la arquitectura se diseñaron y construyeron los circuitos impresos, se soldaron los componentes y los diferentes sensores para medir los datos. Además se diseñó una estructura mecánica y llantas que se imprimieron en una impresora 3D la cual conformaría el vehículo tipo rover. Gracias al excelente trabajo realizado se obtuvo el primer lugar en el Cuarto Concurso Nacional de PicoSatélites Educativos CanSat en la categoría come back.
\end{abstract}

CanSat, Modelo V, vehículo rover

\begin{abstract}
It is described how a CanSat pico-satellite type rover was designed and built to compete in the Fourth CanSat National Educational Satellites Competition. Students of Engineering in Information Technologies and Communications with the support of teachers of that career designed and built the pico-satellite called EagleSat V2.0. Using the methodology of the "V" model, the mission was conceptualized which was the sending of data of: internal and external temperature, pressure, relative humidity, altitude, longitude, latitude, battery level, vibration and acceleration through telemetry to an earth station; take video and return to the starting point using a rover type vehicle; the requirements and architecture of all stages of the EagleSatV2.1 were specified. Starting from the architecture, the printed circuits were designed and built, the components and the different sensors were welded to measure the data. In addition, a mechanical structure and tires were designed and printed on a $3 \mathrm{D}$ printer which would make up the Rover type vehicle. Thanks to the excellent work done, the first place was obtained in the Fourth CanSat National Satellites Educational Contest in the comeback category.
\end{abstract}

CanSat, method in V, rover vehicle

Citación: MANCILLA-CEREZO, Josue, PALACIOS-GARCIA, Ana Cristina, PÉREZ-LOPEZ, Adriana y JIMENEZGONZALEZ, Diane Grace. Diseño y construcción de un pico-satélite educativo CanSat tipo rover denominado EagleSat V2.1. Revista de Ingeniería Innovativa. 2019. 3-12: 17-23

*Correspondencia al Autor (Correo electrónico: jmc_itst@outlook.es)

$\dagger$ Investigador contribuyendo como primer Autor. 


\section{Introducción}

Fue al final de la década de los noventas cuando el profesor Robert Twiggs, propuso la construcción de un nuevo satélite que cupiera en el volumen de una lata de refresco, al cual se le llamo "CanSat" (Kawashima, 2016). Este picosatélite tiene el objetivo principal de trasmitir los conceptos básicos de diseño y construcción de satélites artificiales entre los estudiantes que lo construyen (Colín et al. 2016).

Existen diversas competencias CanSat entre las cuales destacan las internacionales: ARLISS en Estados Unidos (Sako et al. 2001) y UNISEC en Japón (NASA, 2012). En México destaca la competencia organizada por UNISEC México (University Space Enginnering Consortium), que desde el año 2015 ha organizado los concursos nacionales de picosatélites educativos CanSat (UNISEC, 2018). En el cuarto concurso se abrieron dos categorías: Telemetría y comeback (UNISEC et al, 2018).

El equipo de trabajo de estudiantes y maestros, ha participado en tres de los concursos nacionales de pico-satélites educativos CanSat. La primera participación fue en el segundo concurso, en el cual se participó con el picosatélite EagleSat en la categoría telemetría (Sombrerero et al, 2016) y se obtuvo el primer lugar (Macilla et al., 2017).

La segunda participación fue en el tercer concurso, donde se compitió con dos CanSat: WashiSat (Mancilla, Palacios, Pérez y Torija, 2018) y EagleSat V2.0 (Mancilla et al., 2018) en las categorías telemetría y comeback, respectivamente, en las cuales se obtuvo los primeros lugares.

También se participó en el cuarto concurso con el pico-satélite WashiSat V1.0 en la categoría telemetría (Mancilla, Palacios, García y Vázquez, 2019). Y con el pico-satélite EagleSat V2.1 en la categoría comeback.

\section{Descripción del método}

Siguiendo la metodología del diagrama en "V", que indica que las fases a seguir son: prefase, fase $A$, fase $B$, fase $C$, fase $D$, fase $E$ y fase $F$ (Bermúdez, 2016), se diseñó y fabricó el CanSat tipo rover EagleSat V2.1.
1. Prefase: estudio conceptual y selección de misión a desarrollar.

La motivación que se tuvo para la selección de esta misión fueron los diferentes rover planetarios que se han lanzado para el estudio de la luna y el planeta marte desde la década de los setenta hasta la actualidad, entre los que se encuentran:

Lunokhod. Primer robot rover de control remoto en la luna (Kassel, 1971).

Sojourner. Primer rover usado para moverse autónomamente en marte (NASA, 1997).

- $\quad$ Spirit y opportunity. Enviados a marte en el verano de 2003 para una misión conjunta. Su misión fue la exploración de las variedades de rocas y conocer la historia del agua en ese planeta (Leger et al, 2015).

- Curiosity. Es el sucesor de los rover mencionados anteriormente. Su misión comenzó el 11 de noviembre de 2011, esta misión consiste en recabar datos y muestras del cráter llamado "Gale Crater" en marte (Benowiz, 2014)

InSight. Su misión consiste en investigar la composición interna del planeta marte utilizando un taladro para analizar muestras del subsuelo marciano (Good y Johnson, 2019).

- ExoMars 2020. Cuyo lanzamiento está previsto para julio o agosto de 2020 y su aterrizaje en Marte para febrero de 2021, tendrá un total de 23 cámaras que crearán amplias fotografias panorámicas y será lanzado por la agencia espacial europea (Forssmann, 2017).

- Con base a la motivación se decidió que la misión fuera el envío de datos de: temperatura interna y externa, presión, humedad relativa, altitud, longitud, latitud, nivel de batería, vibración y aceleración por medio de telemetría a una estación terrena; tomar video y el retorno al punto de partida mediante un vehículo tipo rover.

2. Fase A: desarrollo de los conceptos y tecnología a utilizar.

Los requerimientos de la misión se presentan en la Tabla 1. 


\begin{tabular}{|c|c|c|}
\hline $\begin{array}{c}\text { Requerimientos del } \\
\text { sistema }\end{array}$ & $\begin{array}{l}\text { Subsistemas } \\
\text { comprendidos }\end{array}$ & Metas \\
\hline $\begin{array}{l}\text { Debe tener dimensiones } \\
\text { de } 6.5 \mathrm{~cm} \text { de diámetro y } \\
20 \mathrm{~cm} \text { de largo. }\end{array}$ & \begin{tabular}{|l|} 
Potencia, \\
computadora, \\
misión, \\
$\begin{array}{l}\text { comunicación } \quad y \\
\text { chasis. }\end{array}$
\end{tabular} & $\begin{array}{lr}\text { Diseñar } & \text { una } \\
\text { arquitectura compacta. }\end{array}$ \\
\hline $\begin{array}{l}\text { Masa máxima del } \\
\text { CanSat } 1 \mathrm{Kg} \text {. }\end{array}$ & $\begin{array}{l}\text { Potencia, } \\
\text { computadora, } \\
\text { misión, } \\
\text { comunicación } \\
\text { chasis. }\end{array}$ & \begin{tabular}{|lrr} 
Diseñar & sistemas \\
esbeltos, & & \\
seleccionando & \\
materiales & & y \\
dispositivos & de & bajo \\
peso. & & \\
\end{tabular} \\
\hline $\begin{array}{lr}\text { Alimentación } & \text { del } \\
\text { CanSat } & \text { será } \\
\text { suministrada } & \text { por } \\
\text { batería. } & \end{array}$ & Potencia. & $\begin{array}{l}\text { Selección de una } \\
\text { batería que sea capaz } \\
\text { de suministrar energía } \\
\text { por lo menos } 30 \\
\text { minutos. }\end{array}$ \\
\hline $\begin{array}{l}\text { La batería debe ser de } \\
\text { fácil acceso. }\end{array}$ & Chasis. & $\begin{array}{l}\text { Diseñar este } \\
\text { subsistema de modo } \\
\text { que el cambio de } \\
\text { batería no afecte a los } \\
\text { demás subsistemas. }\end{array}$ \\
\hline $\begin{array}{l}\text { El CanSat debe tener un } \\
\text { interruptor principal en } \\
\text { un lugar accesible. }\end{array}$ & Chasis. & $\begin{array}{l}\text { Debe tener el botón de } \\
\text { encendido y apagado } \\
\text { en un lugar de fácil } \\
\text { acceso. }\end{array}$ \\
\hline $\begin{array}{ll}\text { Sistema } & \text { de } \\
\text { recuperación. } & \end{array}$ & Recuperación. & $\begin{array}{lr}\text { Construir } & \text { un } \\
\text { paracaídas } & \text { que } \\
\text { asegure } & \text { la } \\
\text { recuperación } & \text { óptima } \\
\text { del CanSat. } & \\
\end{array}$ \\
\hline $\begin{array}{l}\text { Velocidad del descenso } \\
\text { entre } 5 \text { y } 12 \mathrm{~m} / \mathrm{s} \text {. }\end{array}$ & Recuperación. & $\begin{array}{l}\text { Diseñar el paracaídas } \\
\text { para que descienda a } 5 \\
\mathrm{~m} / \mathrm{s} \text {. }\end{array}$ \\
\hline $\begin{array}{l}\text { Alcance de radio entre } \\
400 \text { y } 500 \text { metros. }\end{array}$ & Comunicación. & $\begin{array}{l}\text { Seleccionar } \quad \text { un } \\
\text { dispositivo capaz de } \\
\text { mantener } \\
\text { comunicación durante } \\
\text { el descenso. }\end{array}$ \\
\hline Sistema rover & $\begin{array}{l}\text { Potencia, } \\
\text { computadora, } \\
\text { misión, } \\
\text { comunicación } \\
\text { chasis. }\end{array}$ & 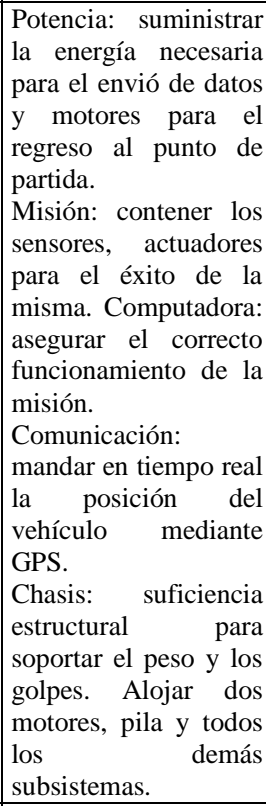 \\
\hline $\begin{array}{lr}\text { Medición } & \text { de } \\
\text { temperatura } & \text { (externa e } \\
\text { interna), presión, } \\
\text { humedad relativa, } \\
\text { latitud, longitud, altitud, } \\
\text { aceleración, vibración, } \\
\text { nivel de batería y video. }\end{array}$ & $\begin{array}{l}\text { Potencia, misión, } \\
\text { comunicación y } \\
\text { computadora. }\end{array}$ & $\begin{array}{l}\text { Potencia: debe tener el } \\
\text { voltaje necesario para } \\
\text { todos los dispositivos } \\
\text { y espacio para } \\
\text { alimentar la cámara. } \\
\text { Misión: debe albergar } \\
\text { todos los sensores. } \\
\text { Comunicación: debe } \\
\text { albergar espacio para } \\
\text { el GPS. } \\
\text { Computadora: debe } \\
\text { contar con un } \\
\text { dispositivo } \\
\text { programable. }\end{array}$ \\
\hline
\end{tabular}

Tabla 1 Requerimientos de la misión Fuente: Elaboración Propia
Con base a los requerimientos se seleccionaron los dispositivos necesarios para cumplir las metas, estos dispositivos se mencionan en la Tabla 2.

\begin{tabular}{|c|c|}
\hline Actividad & Dispositivo \\
\hline $\begin{array}{lrr}\text { Medir } & & \text { Temperatura } \\
\text { externa } & \text { y } & \text { humedad } \\
\text { relativa } & & \\
\end{array}$ & DHT 11 \\
\hline $\begin{array}{l}\text { Medir Presión, altitud, } \\
\text { vibración, aceleración y } \\
\text { temperatura interna }\end{array}$ & GY-88 \\
\hline Medir Longitud y Latitud & GPS GY-NEO6MV2 \\
\hline Tomar Video & Mini cámara espía \\
\hline $\begin{array}{l}\text { Computadora y envió de } \\
\text { datos }\end{array}$ & $\begin{array}{l}\text { Arduino pro mini y Xbee } \\
\mathrm{S} 2\end{array}$ \\
\hline Vehículo rover & $\begin{array}{l}\text { Diseño de chasis y llantas, } \\
\text { impresión 3D, motores y } \\
\text { controlador L298N }\end{array}$ \\
\hline \begin{tabular}{|ll} 
Dispositivo para \\
desprender paracaídas
\end{tabular} & Mini servo motor \\
\hline
\end{tabular}

Tabla 2 Dispositivos seleccionados

Fuente: Elaboración Propia

3. Fase B: diseños preliminares de arquitectura para cumplir la misión

Una vez seleccionados los dispositivos se inició con los bosquejos preliminares de lo que sería la arquitectura del EagleSat V2.1. Los bosquejos generales de las etapas se muestran en las Figura 1, donde se puede apreciar las medidas que tendrían las etapas que irían montadas en el chasis y la distribución de algunos componentes y en la Figura 2 se muestra como sería el EagleSat V2.1 cuando se terminara de construir.

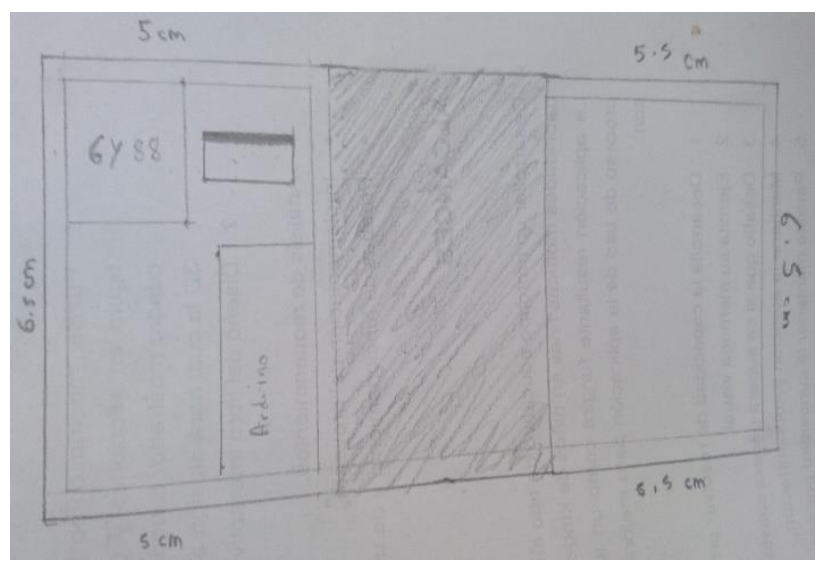

Figura 1 Bosquejo de las etapas y medidas Fuente: Elaboración Propia 


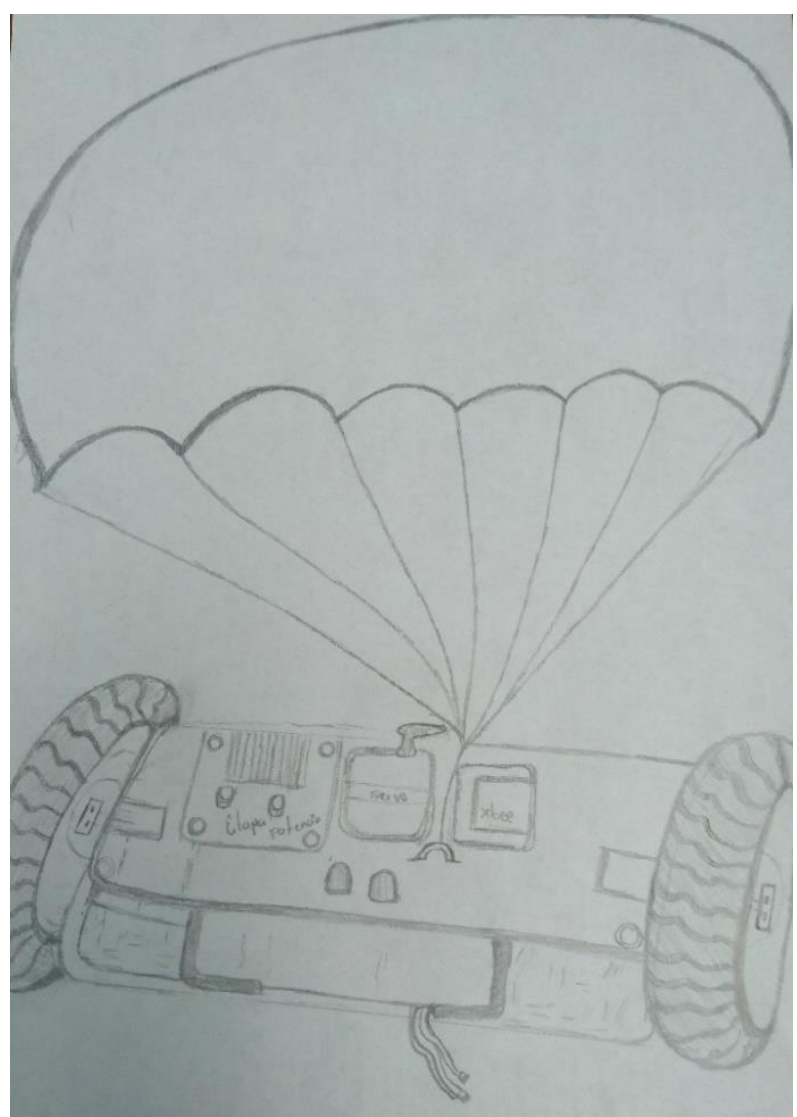

Figura 2 Bosquejo EagleSat V2.1 final Fuente: Elaboración Propia

\section{Fase C: diseños finales y fabricación.}

Siguiendo los bosquejos y las ideas generadas en la Fase B se realizaron los diseños electrónicos los subsistemas así como el diseño mecánico del chasis. Los diseños electrónicos se muestran en la Figura 3, se diseñaron dos etapas que contuvieron los sensores. El diseño del chasis se puede observar en el Figura 4, este chasis cuenta con las cavidades donde podrán ser contenidas la batería, la cámara de video, el sensor DHT 11 y los motores.

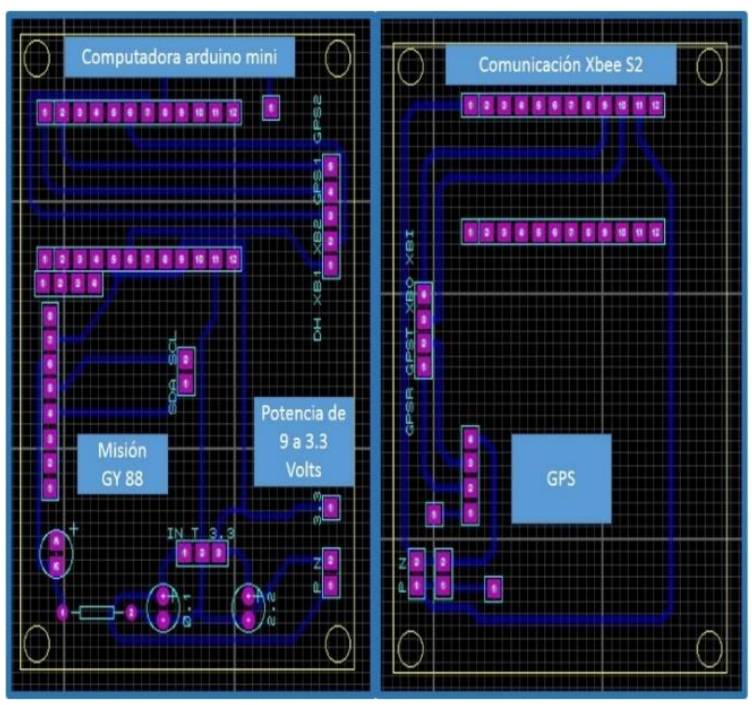

Figura 3 Diseños electrónicos de las etapas Fuente: Elaboración Propia

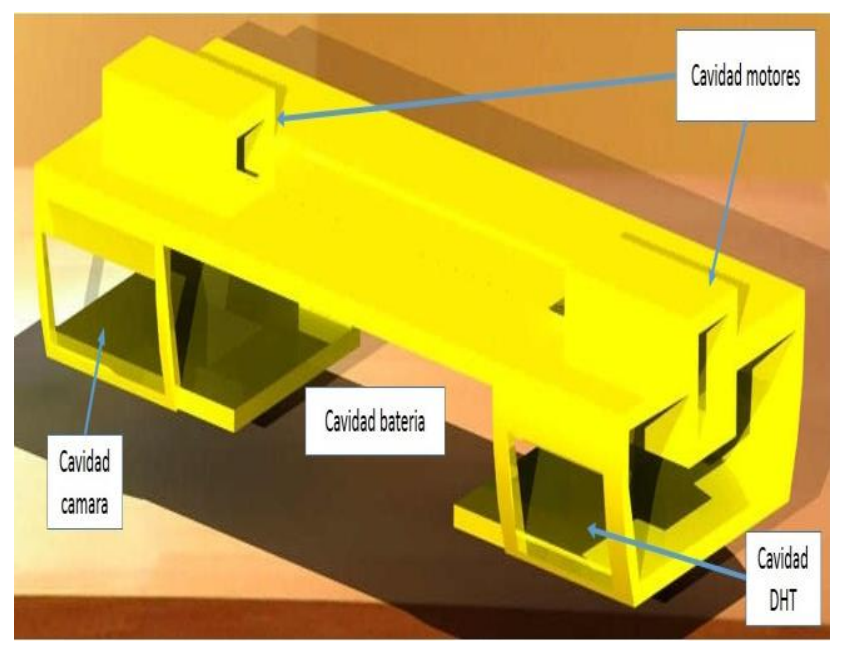

Figura 4 Diseño final chasis Fuente: Elaboración Propia

También se diseñaron llantas de acuerdo a las necesidades del CanSat y se eligieron unas llantas helicoidales, en la Figura 5 se puede apreciar el diseño de estas llantas.

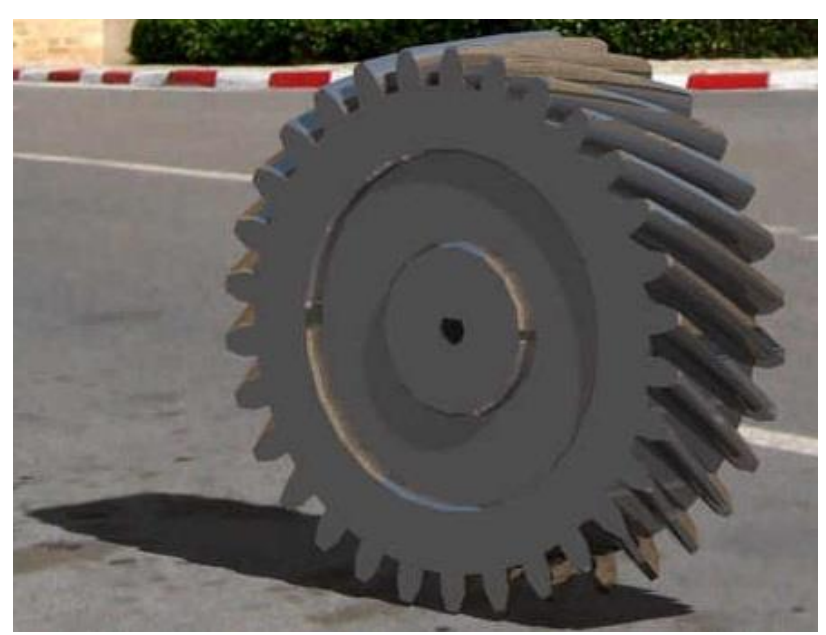

Figura 5 Diseño final llanta

Fuente: Elaboración Propia

Posteriormente, se comenzó la fabricación de las etapas electrónicas por el método de planchado para realizar los circuitos impresos y a la par se imprimieron los diseños del chasis y las llantas utilizando una impresora 3D como se puede apreciar en las Figuras 6 y 7. 


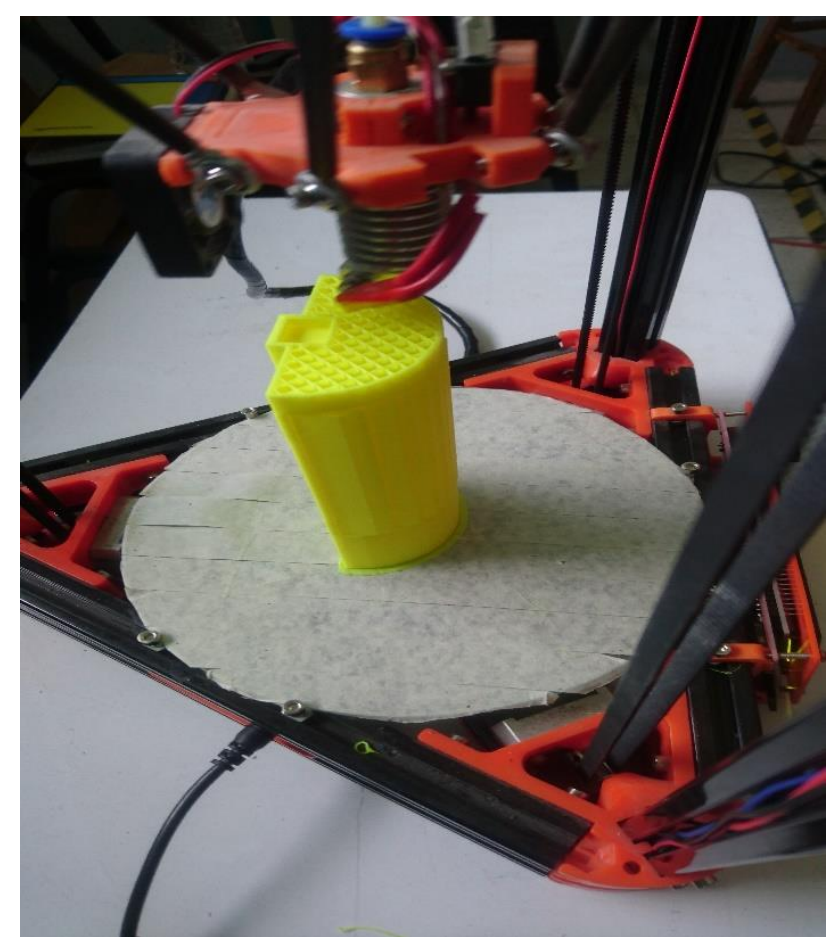

Figura 6 Impresión 3D chasis Fuente: Elaboración Propia

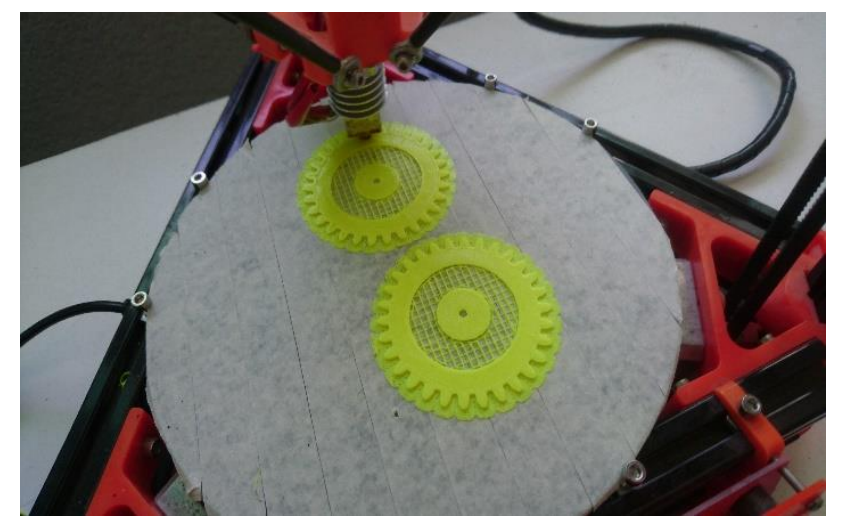

Figura 7 Impresión 3D llantas Fuente: Elaboración Propia

\section{Fase D: Integración.}

Una vez construidas las etapas se realizaron pruebas de integración cumpliendo con los requerimientos y de funcionamiento de las etapas integrándolas e interconectándolas para comprobar su correcto desempeño. La prueba de integración entre llanta y motor así como del tamaño de diámetro requerido se muestra en la Figura 8.

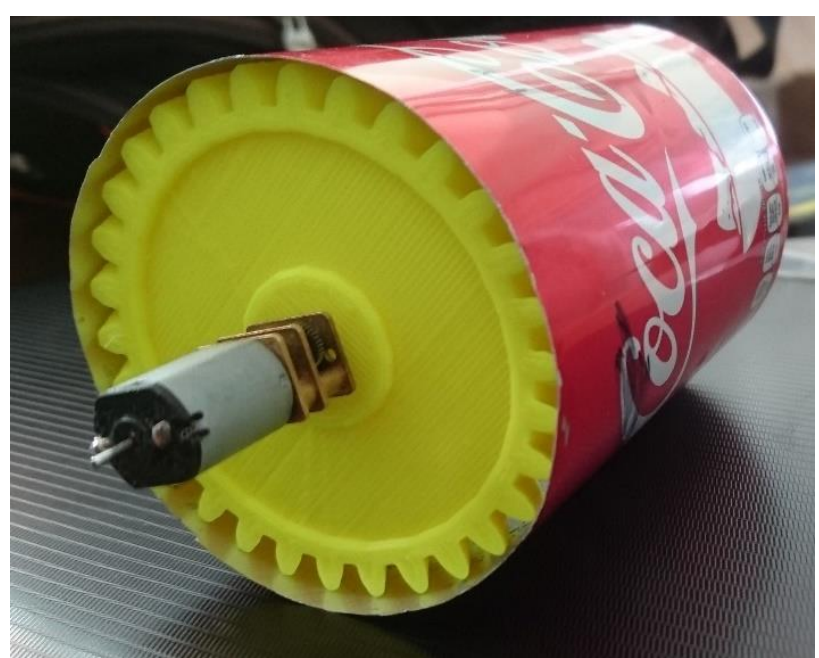

Figura 8 Prueba de integración motor-llanta y tamaño Fuente: Elaboración Propia

En la Figura 9 se muestran los datos recibidos: aceleración y giro en los 3 ejes (x, y, z), altitud y presión atmosférica; del sensor gy 88 , en prueba de funcionamiento de los subsistemas.

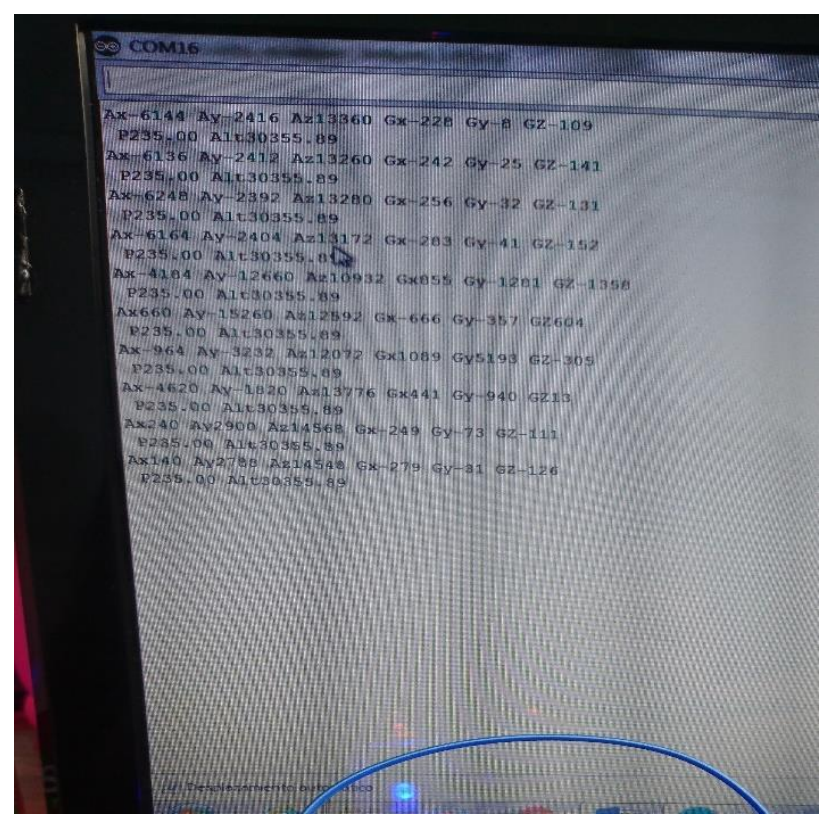

Figura 9 Prueba subsistemas

Fuente: Elaboración Propia

\section{Fase E: Sistema}

Se integraron las etapas electrónicas con el chasis y las llantas para conformar el picosatélite EagleSat V2.1 el cual se puede apreciar en la Figura 10. 


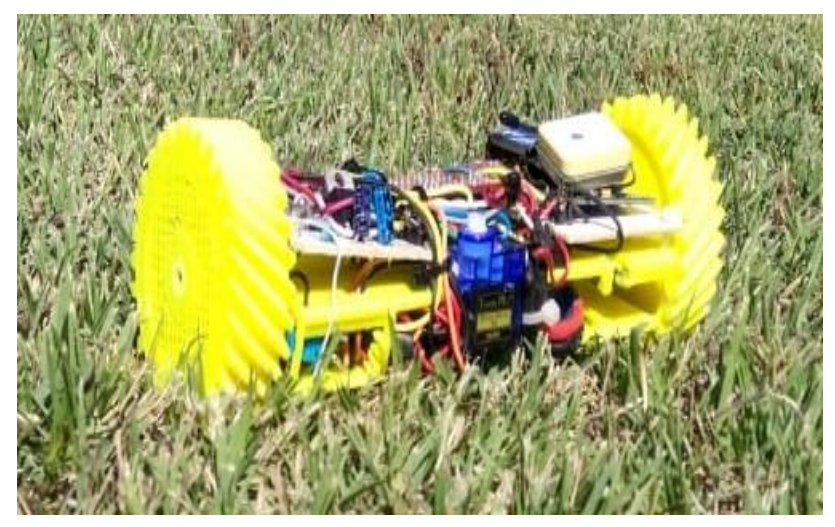

Figura 10 Pico-satélite EagleSat V2.1

Fuente: Elaboración Propia

\section{Resultados}

\section{Fase F: Misión.}

Se diseñó y construyó el pico-satélite EagleSat V2.1, con el cual se participó en el Cuarto Concurso Nacional de Pico-Satélites Educativos CanSat en la categoría de Comeback, en el cual orgullosamente se obtuvo el primer lugar. En la Figura 11, se puede apreciar a los estudiantes exponiendo su CanSat ante los jueces del concurso. Así mismo la Figura 12 se presenta el primer lugar del EagleSat V2.1.

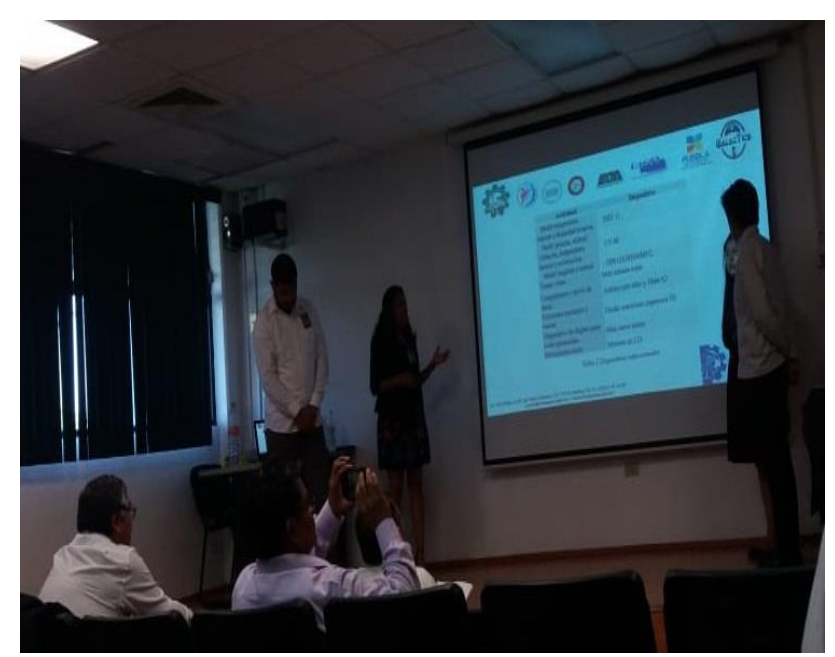

Figura 11 Exposición ante jueces Fuente: Elaboración Propia

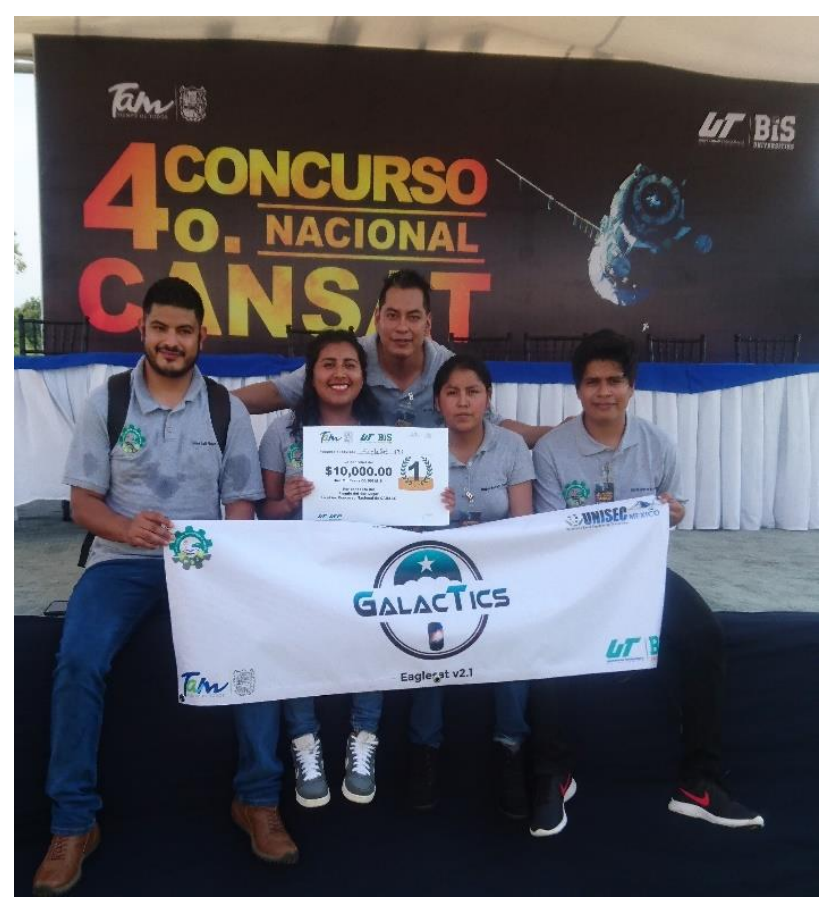

Figura 12 Primer lugar categoría comeback Fuente: Elaboración Propia

\section{Agradecimiento}

Se agradece al maestro Eliseo Ramírez Torres y a todo el personal del ITST por el financiamiento en el desarrollo de este CanSat.

\section{Conclusiones}

Existe un conflicto con respecto a la velocidad de los motores, ya que muchas veces no giran a las mismas revoluciones por segundo y esto ocasiona que el vehículo no avance y solo gire en su propio eje, por lo cual se recomienda usar encoders o algún otro dispositivo de censado para garantizar que los motores giren a la misma velocidad.

Otro conflicto ocurre cuando los motores van montados directamente al chasis, esto hace que el chasis de vueltas y pone en riesgo la integridad de los subsistemas, este conflicto puede solucionarse poniendo un llanta loca como soporte pero le quita movilidad al vehículo.

La metodología del diagrama en "V" asegura el éxito de una misión CanSat, siempre y cuando sea llevado acabo paso por paso, sin suponer o dar avances por hechos. 
Los alumnos que adquieren las competencias básicas en el diseño y construcción de satélites, construyendo un CanSat son más competentes cuando se les presentan proyectos similares, muestra de ello son dos alumnas que a la fecha están realizando residencia profesional en el Instituto Nacional de Astrofísica Óptica y Electrónica, desarrollando proyectos en esta área.

\section{Referencias}

Benowiz, E. (2014). The curiosity mars rover's fault protection engine, IEEE International Conference on Space Mission Challenges for Information Technology. Jet propulsion Laboratory, California institute of technology.

Bermúdez, B. (2016). Cansat: lata-satélite. CiENCiA UANL. 19, (81), p. 71-75.

Colín, A., et al. (2016). Construcción de un picosatélite cansat. CiENCiA UANL. 19, (81), p. 34-38.

Forssmann, A. El rover de la futura misión Mars 2020 tendrá 23 cámaras. National Geographic, España.

Good, A. y Johnson, A. (2019). InSight's Team Tries New Strategy to Help the Mole, NASA. Pasadena, California.

Kassel, S. (1971), Lunokhod-1 Soviet Lunar Surface Vehicle. Advanced Research Projects Agency. DAHC15 67 C 0141.

Kawashima, R. (2016). CanSat Leader Training Program -Past, Present and Future-. CiENCiA UANL. 19, (81), p. 76-82.

Leger et al. (2015), Mars Exploration Rover Surface Operations: Driving Spirit at Gusev Crater. Proc. IEEESMC, Waikoloa, HI.

Mancilla, J., Martínez, R. M., Palacios, A. C., y Hernández, L. R. (2017). Diseño y construcción de un pico satélite cansat denominado EagleSat. Aplicación del Saber: Casos y Experiencias, 3(1), 1733-1738.

Mancilla, J., Palacios, A. C., García, L. A., y Vázquez, N. I. (2019). Diseño y construcción de un pico-satélite CanSat como herramienta para la formación de ingenieros. Revista electrónica ANFEI digital, por publicar.
Mancilla, J., Palacios, A. C., Hernández, L. R., y de la Cruz, G. J. (2018). Diseño y construcción de un pico satélite cansat tipo rover denominado EagleSat V2.0. Aplicación del Saber: Casos y Experiencias, 4(1), 1272-1277.

Mancilla, J., Palacios, A. Pérez, B., y Torija, E. Diseño y construcción de un pico-satélite educativo CanSat denominado WashiSat. Revista de Tecnologías Computacionales. 2, (7), p. 1-7. Disponible en: http://ecorfan.org/taiwan/research_journals/Tec nologias_Computacionales/vol2num7/Revista de_Tecnolog\%C3\%ADas_Computacionales_V 2_N7_1.pdf

NASA. (2012). Space Flight Program and Project Management Requirements. National Aeronautics and Space Administration, California. Technical TP-2014-216648. NPR 7120.5. Disponible en: http://nodis3.gsfc.nasa.gov/npg_img/N_PR_71 20_005E_/N_PR_7120_005E_.pdf

Sako, N., et al. (2001). Cansat suborbital Launch Experiment-University Educational Space using picosatellite. Acta Astronáutica, 48, (15), p.767776.

UNISEC México

www.unisecmexico.com

Sombrerero, J., et al. (2016). Sonda espacial. CiENCiA UANL. 19, (81), p. 40-44.

XIV. NASA (1997). Mars Pathfinder. NASA Facts. p. 1-8.

UNISEC, et al (2018). Convocatoria: 4to. Concurso Nacional de Pico-Satélites Educativos CANSAT. Disponible en: http://www.unisecmexico.com/ 